\title{
Comparative seismology of pre- and main sequence stars in the instability strip
}

\author{
M. Suran ${ }^{1}$, M. Goupil ${ }^{2}$, A. Baglin ${ }^{3}$, Y. Lebreton ${ }^{2}$, and C. Catala ${ }^{4}$ \\ 1 Astronomical Institute of Romanian Academy \\ 2 DASGAL, UMR CNRS 8633, Observatoire de Paris-Meudon, France \\ 3 DESPA, UMR CNRS 8632, Observatoire de Paris-Meudon, France \\ ${ }^{4}$ LAT, UMR CNRS 5572, Observatoire de Midi-Pyrénées, France
}

Received 22 January 2001 / Accepted 30 March 2001

\begin{abstract}
Pulsational properties of $1.8 M_{\odot}$ stellar models covering the latest stages of contraction toward the main sequence up to early hydrogen burning phases are investigated by means of linear nonadiabatic analyses. Results confirm that pre-main sequence stars (pms) which cross the classical instability strip on their way toward the main sequence are pulsationally unstable with respect to the classical opacity mechanisms. For both pms and main sequence types of models in the lower part of the instability strip, the unstable frequency range is found to be roughly the same. Some non-radial unstable modes are very sensitive to the deep internal structure of the star. It is shown that discrimination between pms and main sequence stages is possible using differences in their oscillation frequency distributions in the low frequency range.
\end{abstract}

Key words. stars: oscillations, stars: pre-main sequence

\section{Introduction}

In the vicinity of the main sequence, the HR diagram is confusing as stars of similar global properties but different stages of evolution lie at the same position. In some cases the young pre-main sequence stars are recognized through specific characteristics, for instance the presence of nebulosity or high degree of activity. But, this is not always the case. An alternative is to take advantage of seismological information whenever possible. We therefore carry out a comparative study of the pulsational behavior of pre-main sequence (hereafter pms) and main sequence (hereafter postZams) stars of intermediate mass, in the classical instability strip.

Our study is restricted to the latest stages of the premain sequence contraction when traces of the formation phases have already disappeared, so that a pms model can be built with the quasi-static approximation.

The outer layers of pms and postZams stars having almost the same effective temperature and gravity are very similar. As these layers drive the pulsation in this range of temperature, it is reasonable to expect that pms stars in the instability strip are also destabilized by classical opacity mechanisms and that the same type of modes as for the postZams stars are excited. This idea has been confirmed

Send offprint requests to: M. Goupil, e-mail: goupil@obspm.fr by Suran (1998) and in a more detailed work but for pure radial modes of very young and cool stars by Marconi \& Palla (1998). This supports vibrational instability as an explanation for the observed periodic variations detected for a few stars which are suspected to be in the pre-main sequence stage (Kurtz \& Marang 1994; Catala et al. 1997; Kurtz 1999).

Differences, on the other hand, exist between the two stages: whereas pms has some relics of its gravitational contraction phase, the main sequence nuclear burning modifies the inner core structure, and postZams models develop chemical inhomogeneities. In a preliminary work, Suran (1998) has stressed some consequences of these differences in the structures of the frequency distributions. The present paper further investigates and compares the pulsational properties of these two phases for non-radial oscillations. It is organised as follows: stellar modelling and its physical inputs are described in Sect. 2, where comparable pms and postZams models are selected. Oscillation frequencies associated with nonradial modes for both types of objects are compared in Sects. 3 and 4, and emphasis is put on the structure of the frequency spectra in both stages. Finally in Sect. 5, we discuss the possibility to infer the evolutionary stage, i.e. the age of a variable object in this region of the HR diagram, through the pattern of its frequency spectrum. 


\section{Static models and evolutionary sequences}

\subsection{Evolutionary tracks}

In the early stages of pms evolution, various rapid- dynamical and thermal-processes occur which modify the internal structure of the protostar. By the time the protostar reaches the classical instability strip on its way toward the main sequence, these complex phenomena have long disappeared and the evolution has considerably slowed down. The usual quasi-static approximation then holds and is assumed here. Rotation is not considered and the star is modelled in a spherically symmetric equilibrium state.

Equilibrium models have been computed with CESAM (Morel 1997), an evolutionary code with a numerical accuracy of first order in time and third order in space. The models are built with the fast version of the code i.e. 300 mesh points in mass; pulsation calculations on the other hand use an extended 2000 mesh grid, calculated from the static model by means of the basis of the spline solution.

The EFF equation of state (Eggleton et al. 1973) is used as it is sufficient for our purpose in this mass range. Opacities are provided by the Livermore Library (Iglesias \& Rogers 1996), complemented at low temperatures $(T \leq 10000 \mathrm{~K})$ by Alexander \& Ferguson 's (1994) tables. The nuclear network contains the following species ${ }^{1} \mathrm{H},{ }^{3} \mathrm{He},{ }^{4} \mathrm{He},{ }^{12} \mathrm{C},{ }^{13} \mathrm{C},{ }^{14} \mathrm{~N},{ }^{15} \mathrm{~N},{ }^{16} \mathrm{O},{ }^{17} \mathrm{O}$. The main nuclear reactions of $\mathrm{pp}+\mathrm{CNO}$ cycles are included with the species ${ }^{2} \mathrm{H},{ }^{7} \mathrm{Li},{ }^{7} \mathrm{Be}$ set at equilibrium. Nuclear reaction rates are taken from Caughlan \& Fowler (1988); weak screening is assumed. The isotopic helium ratio is fixed at $\mathrm{He}^{3} / \mathrm{He}^{4}=1.01 \times 10^{-4}$ and the mixture of heavy elements is solar (Grevesse \& Noels 1993). In the convection zones, the temperature gradient is computed according to the standard mixing-length theory, with the mixing-length defined as $l \equiv \alpha H_{\mathrm{p}}$, where $H_{\mathrm{p}}$ is the pressure scale height. Assuming a constant value of $\alpha$ along the main sequence (Fernandes et al. 1998), we have chosen $\alpha=1.67$, the value obtained for a calibrated Sun computed with the same physical assumptions. The atmospheric layers are treated with the Eddington's grey approximation.

We consider the lower part of the instability strip where one encounters the group of $\delta$ Scuti stars defined here as Pop I stars in a postZams stage. The associated mass range approximately is $1.6-2 M_{\odot}$. A mass of $1.80 M_{\odot}$ with a standard chemical composition for Population I $(X=0.71, Y=0.27, Z=0.02)$ is therefore chosen as typical for the present work.

Figure 1 shows the evolutionary track running from the latest pms stages to the early postZams ones for this $1.80 M_{\odot}$ stellar model. Peculiar stages are indicated and their characteristics listed in Table 1. Local maxima of the luminosity along the pms track correspond to the onset of rapid nuclear burning stages: ignition of the $p-p$ reaction occurs close to models M6 and M7; the $\mathrm{C}^{12} \mathrm{~N}^{14}$ transformation (close to models M12, M13 and M14) gives rise to core convection. As the CNO cycle contribution becomes

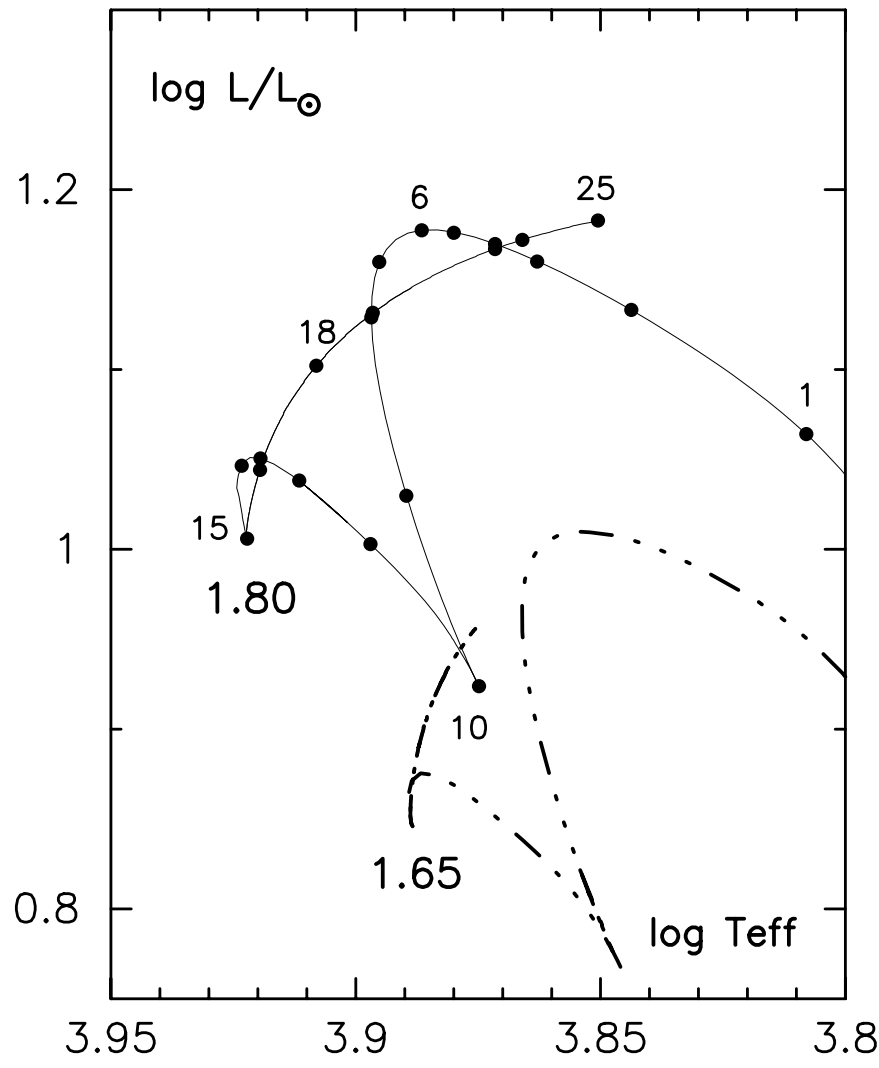

Fig. 1. Evolutionary sequence of a $1.80 M_{\odot}$ model (solid line). Selected models listed in Table 1 are indicated by dots and labeled on the track. A $1.65 M_{\odot}$ evolutionary sequence is also shown for comparison (dashed line).

increasingly important and eventually dominant, the convective core slightly expands till the stage of M10. Once the M14 stage is reached, the star definitely settles into the CNO cycle burning phase. The zero age main sequence (Zams) model is defined as the model with the minimum luminosity just before evolution starts back toward the red. At this stage, M15 in Table 1, the gravitational energy $\left(\epsilon_{\text {grav }}\right)$ represents less than $1 \%$ of the total generated energy $\left(\epsilon_{\text {tot }}\right)$ and the central initial hydrogen content has been only slightly modified (see Table 1).

This study does not include the earliest stages, hence it cannot provide exact values of ages. However, evolutionary time scales for phases after the ignition of the central hydrogen burning phase are reliable. We have therefore arbitrarily chosen the stage M1 as the initial epoch.

\subsection{Structural differences between pms ans postZams models with same global properties}

As mentionned earlier, a source of ambiguity comes from the fact that pms and postZams stars have similar surface properties and therefore lie at the same position in a HR diagram. For instance, our $1.8 M_{\odot}$ sequence in Fig. 1 shows that the three pms models M4, M8, M13 can be confused with the three postZams models M23, M19, M16 respectively, on the basis of their sole location 
Table 1. Selected stellar models from the $1.80 M_{\odot}$ evolutionary sequence. The second column gives the effective temperature $T$ of the model. Ages are in Myr, luminosities $L$ and total stellar radii, $R$, in solar units, convective radii $r_{\text {conv }}$ in unit of total stellar radii. $X_{\mathrm{c}}$ is the central hydrogen content in percentage.

\begin{tabular}{|l|l|l|l|l|l|l|}
\hline & $\log T$ & $\log L$ & $R$ & age & $X_{\mathrm{c}}$ & $r_{\text {conv }}$ \\
\hline M1 & 3.808 & 1.064 & 2.751 & .0 & 0.7143 & 0.000 \\
M2 & 3.844 & 1.133 & 2.527 & .4 & 0.7143 & 0.000 \\
M3 & 3.863 & 1.160 & 2.389 & .6 & 0.7142 & 0.000 \\
M4 & 3.872 & 1.170 & 2.319 & .7 & 0.7142 & 0.068 \\
M5 & 3.880 & 1.176 & 2.251 & .8 & 0.7142 & 0.124 \\
M6 & 3.887 & 1.177 & 2.184 & .9 & 0.7142 & 0.166 \\
M7 & 3.895 & 1.160 & 2.056 & 1.1 & 0.7142 & 0.221 \\
M8 & 3.897 & 1.129 & 1.970 & 1.25 & 0.7142 & 0.243 \\
M9 & 3.890 & 1.030 & 1.816 & 1.6 & 0.7141 & 0.258 \\
M10 & 3.875 & 0.924 & 1.721 & 2.4 & 0.7139 & 0.239 \\
M11 & 3.897 & 1.003 & 1.702 & 3.8 & 0.7136 & 0.211 \\
M12 & 3.912 & 1.038 & 1.657 & 4.4 & 0.7135 & 0.186 \\
M13 & 3.919 & 1.050 & 1.621 & 4.8 & 0.7134 & 0.186 \\
M14 & 3.923 & 1.047 & 1.586 & 5.2 & 0.7134 & 0.201 \\
M15 & $\mathbf{3 . 9 2 2}$ & $\mathbf{1 . 0 0 6}$ & $\mathbf{1 . 5 2 1}$ & $\mathbf{1 1 .}$ & $\mathbf{0 . 7 1 2 0}$ & $\mathbf{0 . 2 0 5}$ \\
M16 & 3.920 & 1.044 & 1.608 & 300. & 0.6383 & 0.204 \\
M17 & 3.918 & 1.058 & 1.649 & 400. & 0.6097 & 0.202 \\
M18 & 3.908 & 1.102 & 1.813 & 700. & 0.5127 & 0.197 \\
M19 & 3.897 & 1.131 & 1.978 & 900. & 0.4355 & 0.191 \\
M20 & 3.893 & 1.139 & 2.029 & 950. & 0.4142 & 0.190 \\
M21 & 3.889 & 1.146 & 2.086 & 1000. & 0.3920 & 0.188 \\
M22 & 3.876 & 1.162 & 2.248 & 1120. & 0.3340 & 0.182 \\
M23 & 3.872 & 1.167 & 2.312 & 1160. & 0.3132 & 0.180 \\
M24 & 3.866 & 1.172 & 2.383 & 1200 & 0.2913 & 0.178 \\
M25 & 3.851 & 1.183 & 2.594 & 1300. & 0.2320 & 0.170 \\
\hline
\end{tabular}

in the HR diagram (Table 1 ). The question we address here is whether small differences in the structures of the pms and postZams models can induce significant changes in their oscillation spectra. The second issue is to determine whether these frequency differences can be used to discriminate between the two evolutionary stages.

Accordingly, our procedure consists of comparing seismological properties of pms and postZams models having the same position in the HR diagram and the same mass (herafter "associated models").

As expected, the outer layers of associated models are very similar: density and temperature profiles and therefore the opacity profile are almost identical, we therefore refrain from showing them.

On the other hand, the central regions significantly differ. A pms model is still slightly contracting and remains chemically homogeneous. In contrast, a postZams model has a core which traces its nuclear history, changes its chemical composition and density, and builds regions with $\mu$ gradients.

Our youngest models correspond to the phase of the beginning of the $p-p$ chain, with a very small expanding convective core. During the main sequence phase, as the star ages, hydrogen is depleted in the central region and the fully mixed convective core receeds with time starting at the Zams stage (approximately M15).

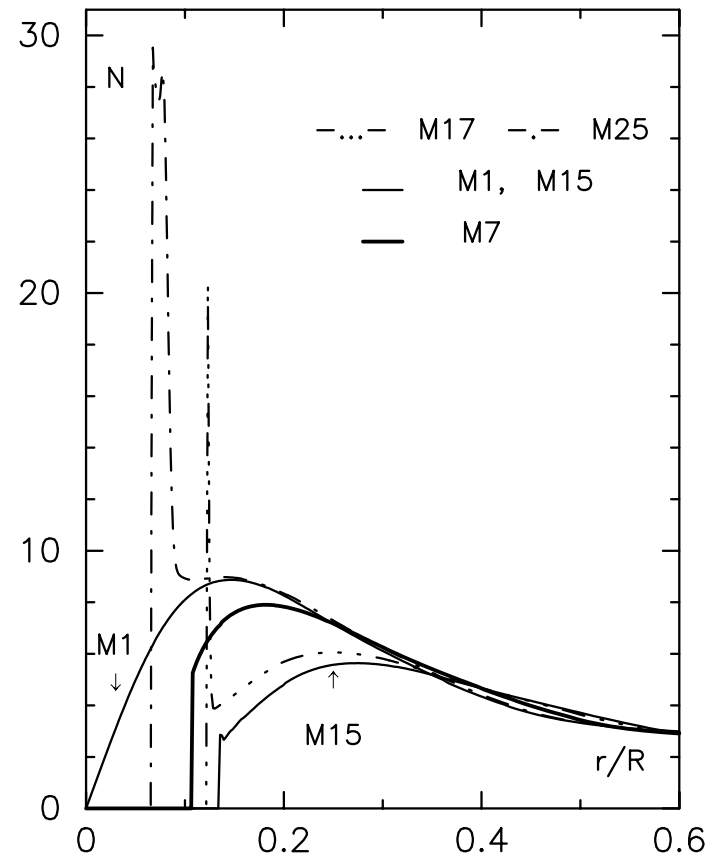

Fig. 2. Behavior of the Brünt-Vaissälä frequency $N$ in normalized unit (see Eq. (1)) as a function of radius (normalized to the total radius) for models along the $1.80 M_{\odot}$ evolutionary sequence: $N$ profiles for two pms models (M1, M7), Zams model (M15) and two postZams models (M17 and M25) are represented.

Signatures of these modifications are clearly seen on the profile of the Brünt Vaissälä frequency, $N$, as a function of radius (Fig. 2). The sharp transition between zero values of $N$ within the convective core and positive values in the overlaying radiative layers delimits the edge of the convective core. As it can be seen in Fig. 2, during the pms evolution, the outward progression of the edge of the expanding convective core can be followed with the correlated outward progression of the inner edge of $N$. The inner $N$ maximum also moves outward and decreases. When the model evolves beyond the Zams on the main sequence, the inner edge and maximum of the BrüntVaissälä frequency receed inwards, while a narrower but higher peak associated with the $\mu$ gradient region forms. It can be expected from Fig. 2 that modes with normalized frequencies (Eq. (1) below) in the range $4-10$ are the most sensitive to the size of the convective core and consequently to the evolutionary status (pms or postZams) of the star.

\section{Nonradial pulsations of pms and postZams models}

At every stage considered here, the time scale of evolution is much longer than the pulsation period. For instance our $1.8 M_{\odot}$ sequence indicates time intervals of about $0.1-1$ Myr between specific stages of evolution represented in Fig. 1. Periods of oscillation of $\delta$ Scuti stars typically range between $1 \mathrm{~h}-3 \mathrm{~h}$, reaching roughly $20 \mathrm{~h}$ for unstable $g$-modes. Growth rates are of the order of 
100-500 yr, still much smaller than evolutionary time scales. The oscillation amplitudes are expected to grow or decay on a much faster (Kelvin-Helmoltz) time scale than the (nuclear) evolutionary time-scale, so that the amplitudes have settled onto their saturating value (limit cycle) long before evolution can have any disturbing influence. These considerations authorize investigations of the pulsations of pms stars by means of usual linear nonadiabatic analyses of static models at a given stage of evolution (Cox 1980; Unno et al. 1989). We treat the nonadiabatic effects in the formulation of Unno et al. (1989), using Suran's oscillation code. The oscillation eigenfrequencies ( $\mathrm{rad} / \mathrm{s})$ are denoted as $\omega$. We will also use frequencies in $\mu \mathrm{Hz}, \nu$, and normalized frequencies, $\sigma$ defined as

$$
\sigma=\frac{\omega}{\sqrt{G M / R^{3}}} .
$$

Frequencies of the modes are labelled in a usual way: $n, l, m$ for the radial order, degree and azimutal number. The radial order of a given mode is determined according to Christensen-Dalsgaard \& Berthomieu (1992) with $n=1$ for the fundamental radial mode. The growth rate is obtained directly from numerical computations but we rather consider the dimensionless $\eta$ parameter (Stellingwerf 1978; Dziembowski 1993):

$$
\eta=\frac{W}{\int_{0}^{1}|w(x)| \mathrm{d} x}
$$

where $x=r / R$ and $W$ is the total mechanical work (Cox 1980; Saio \& Cox 1980)

$$
W=\int_{0}^{1} w(x) \mathrm{d} x=\frac{\pi}{E_{k}} \int_{0}^{M} \frac{P}{\rho} \operatorname{Im}\left\{\left(\frac{\delta P}{P}\right)^{*}\left(\frac{\delta \rho}{\rho}\right)\right\} \mathrm{d} m
$$

where $\delta P, \delta \rho$ are the Lagrangean variations of the pressure and density respectively. The work integral, $W$, is normalized with the total kinetic oscillation energy:

$$
E_{k}=\frac{1}{2} \omega^{2} \int_{0}^{1}|\delta r|^{2} \rho \mathrm{d}^{3} r
$$

where $\delta r$ is the Lagrangean radius variation.

Interaction between pulsation and convection is neglected. This is certainly not justified for the cool stars but the effect is less important for stars close to the Zams. Neglecting this interaction equivalently affects pms and postZams models at the same effective temperature close to the Zams. Hence this cannot significantly affect the main conclusions of the present comparative study.

\subsection{Evolution of the power spectrum with stellar age}

Figure 3 displays the evolution of the normalized frequencies, $\sigma$, for a $1.8 M_{\odot}$ model evolving from stage 1 (pms) to stage 25 (postZams) along the evolutionary track shown in Fig. 1. The plot is restricted to $\ell=0-2$ modes. These modes are usually thought to be the easiest modes to detect, as their visibility coefficients remain large after integration over the whole stellar disk. The radial order interval is chosen such as to cover the range of vibrationally unstable modes. Evolution is represented in abscissae by a quantity which is defined so as to change monotonously throughout the pms and main sequence stages: $\Delta R$ is the difference between the radius of the model, $R$, and the radius of a reference model, $R_{\text {eff }}$, which for obvious reasons is chosen to be the Zams model (M15 model of Table 1). Time goes from left to right. For pms models, $\Delta R=R-R_{\text {ref }} \leq 0$ and for postZams models $\Delta R=R_{\text {ref }}-R \geq 0$.

Normalized frequencies evolve with age quasisymetrically with respect to the Zams stage. Frequencies of radial modes and nonradial pure $p$-modes with given $n, \ell$ remain nearly constant when evolving from late pms to early postZams stages because the models change nearly homologously.

On the other hand, $g$-modes, as they are sensitive to the structure of the inner convective layers, do not behave homologously. One can see in Fig. 3 changes of the frequencies of the $g$-modes which arise from the "breathing" of the core, (which moves back and forth) when a new nuclear reaction starts, at models M4 and M12: frequencies of $g$-modes increase with age for postZams models because the inner maximum of the Brünt-Väissala frequency increases with age and its sharp inner edge moves outward. In a symmetric way with respect to the Zams model, the frequencies of $g$-modes decrease when the pms model evolves toward the Zams stage because the position of the inner maximum of $N$ moves inward with time.

In addition, the well known phenomenon of avoided crossing (Unno et al. 1989) occurs during the latest postZams stages. The development of a $\mu$ gradient at the edge of the convective core of the postZams model causes the development of a sharp peak of the Brünt-Vaissälä frequency. Then nonradial low frequency modes undergo an avoided crossing which shifts the frequency with respect to its value in the absence of avoided crossing. Only those modes which are sensitive to the edge of the convective core have a different behavior before and after the Zams stage. These interacting modes exchange their physical nature. Their frequencies penetrate into the $p$ mode frequency domain, breaking the regularity seen at high frequencies. The progression of this phenomenon into the $p$-mode domain can be followed from mode with $n \sim 1$ $(\Delta R \sim 0)$ up to $n=8(\Delta R \sim 1)$ for $\ell=2$ modes. This leads to different and recognizable patterns in a frequency spectrum, which can be considered as the signature of different structures of the core and can therefore tell whether the star is a pms or a main sequence one.

Finally, frequency calculations show the existence of strange modes i.e. surface modes trapped in very superficial outer layers (Unno et al. 1989; Buchler et al. 1997; Saio et al. 1998). Whether these modes are excited or not is probably strongly dependent on the convectionpulsation interaction not included here. As these surface 

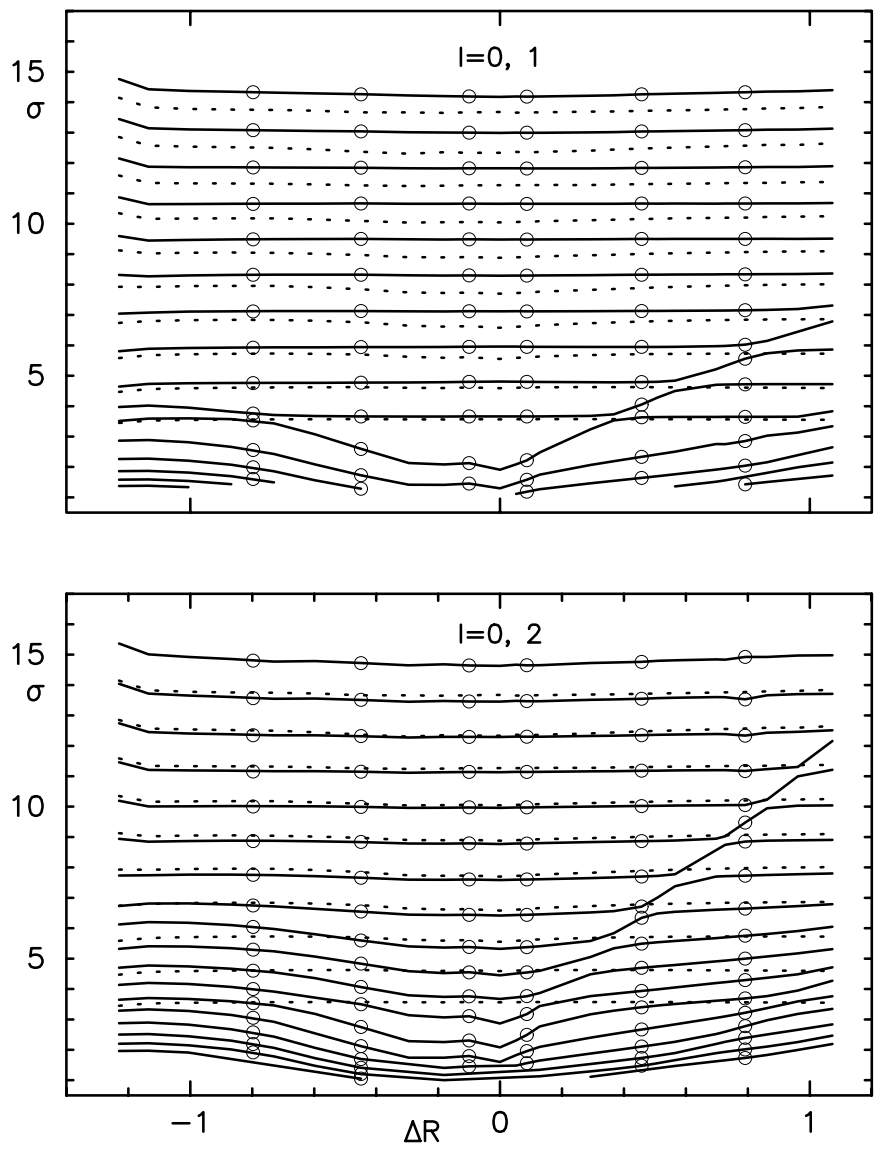

Fig. 3. Normalized frequencies of a $1.8 M_{\odot}$ model evolving from stage 1 to stage 25 of Fig. 1 . Abcissa is $R-R_{\text {ref }}$ for postZams models and $R_{\text {ref }}-R$ for pms models (see text). For sake of clarity, $\ell=1$ et $\ell=2$ are plotted separately: top frame $\ell=0,1$ and bottom frame $\ell=0,2$. Frequencies of radial modes with same radial order are connected with dotted lines; $\ell=1$ and $\ell=2$ are connected with solid lines. Frequencies of associated pms and postZams models M4-M23; M8-M19; M13-M16 are presented with open circles.

modes are not essential for our purpose, they were disregarded in Fig. 3.

\subsection{Vibrational instability}

The linear nonadiabatic calculations for modes with $\ell=$ 0,1,2 for models of Table 1 show that the pms models lying in the classical instability strip are vibrationally unstable. The unstable modes are the same than those for classical $\delta$ Scuti stars, namely low radial order $g$ - and $p$ modes.

Figures 4 shows the behavior of the parameter $\eta$ (Eq. (2)) with increasing normalized frequencies $\sigma$ for $\ell=0,1,2$ modes for the M8 pms and the M19 main sequence models. The instability strength as measured with $\eta$ is independent of the degree $\ell$. The $\eta$ curves for pms and main sequence associated models are almost identical. For these models, modes with radial order in the range $n=1-7$ are found to be unstable.

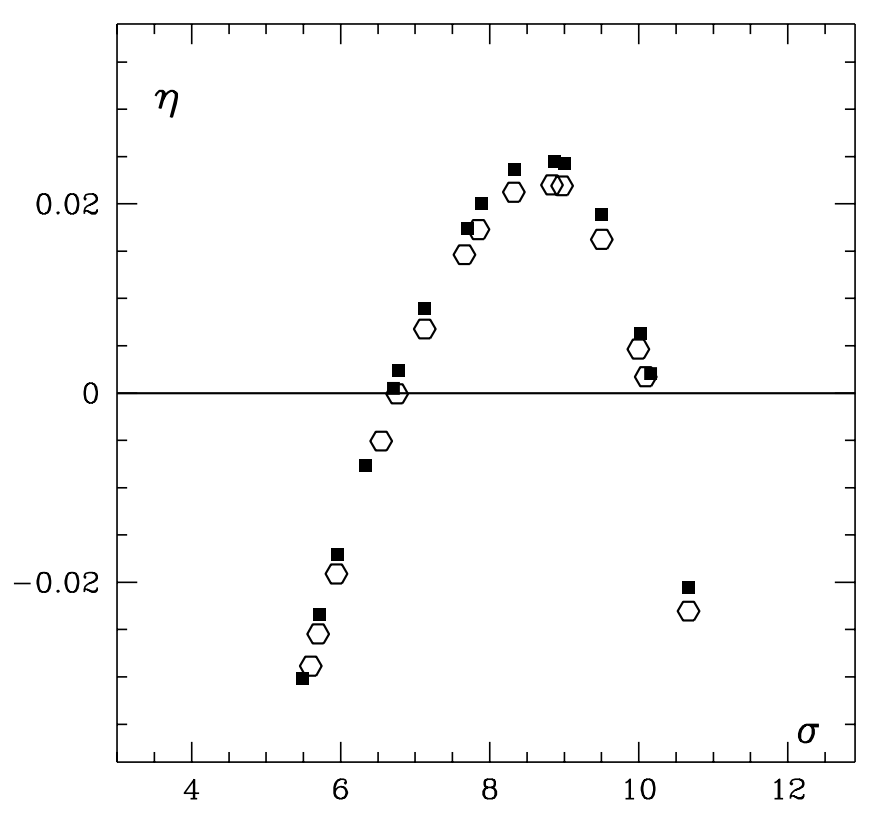

Fig. 4. Growth rate $\eta$ versus normalized frequency $\sigma$ for low radial order $\ell=0,1,2$ modes for the associated pms (M8, open circles) and main sequence (M19, full squares) models.

The work integrands are found to be quite similar for associated pms and postZams models. Computation of the work integrals shows that, for both pms and postZams models, the modes are driven by opacity mechanisms which efficiently operate in the $\mathrm{H}$ and $\mathrm{He}$ ionisation zones. The $\epsilon$ mechanism contributes only very weakly in the deep interior even in the youngest models.

\section{Frequency distributions of pms versus postZams models}

Figure 5 compares frequencies of modes with the same degree $\ell$ for the associated models M4 and M23, represented respectively by solid vertical lines and dashed vertical lines. Frequencies of $\ell=0$ modes with same radial order are nearly identical as shown by the solid lines which nearly coincide with the dashed lines. This is because both models have similar mean density and outer layers. This is also true for the highest frequencies of $\ell=1$ and $\ell=2$ modes which are associated with pure $p$ modes. Differences in the spectral patterns on the other hand exist in the low frequency range. In particular, a few extra frequencies, associated with $\ell=1,2$ modes in avoided crossing, exist for the postZams model. As a consequence, even without a mode identification, a comparison of the spectra for a pms and a postZams models at the same location in the HR diagram tells us whether the star is in a pms or a postZams stage and, in the latter case, yields an indication of its age.

Frequency differences for a given mode between models M4 and M23 reach values as large as $50 \mu \mathrm{Hz}$ in real units. This is emphasized in Fig. 6. Global parameters are identical, therefore radial modes with same radial order have about the same frequencies. The external layers are similar, therefore the excitation range is about the same. 


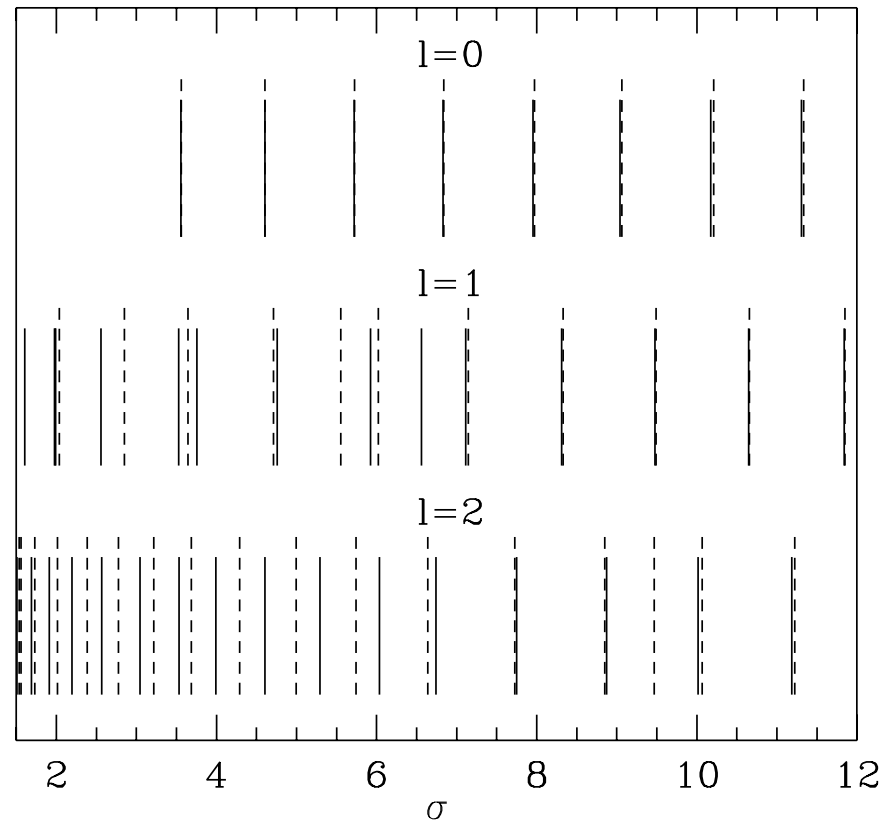

Fig. 5. Frequency distribution for $\ell=0,1,2$ modes for pms M4 (solid lines) and main sequence M23 (dashed lines) models. Ordinate is arbitrary. Abscissa is the normalized frequency $\sigma$.

In this frequency range, $\ell=2$ modes are the most affected by the structure of the deep layers and are the main agents for differences in power spectra.

\section{Toward a seismological diagnostic: Frequency differences}

Pms and postZams models at the same location in the HR diagram have similar mean densities, hence one expects the large separation $\nu_{n, \ell}-\nu_{n-1, \ell}$ to be the same for "associated" models. This is confirmed in Fig. 7 where the large separations for $\ell=0$ modes (for low radial order modes) are displayed for associated models and for the Zams model. The large separation is indeed the same respectively for M4 and M23; for M8 and M19; for M13 and M16. The large separation scales as $\left(G M / R^{3}\right)^{1 / 2}$. Given the mass, the large separation increases with age for pms models (decreasing radii) and decreases with age for postZams models (increasing radii); it is largest for the Zams model which has the smallest radius.

The development of a $\mu$ gradient at the edge of the convective core can be seen on the frequency large separation for nonradial modes in Fig. 8. The large separation for $\ell=2$ modes for associated pms and postZams models is expressed in normalized units and plotted against the normalized frequency $\sigma_{n, \ell=2}$. The existence of a mode in avoided crossing generates a large departure of the frequency difference $\sigma_{n, \ell}-\sigma_{n-1, \ell}$ from its mean value at the frequency of this mode. The frequency at which a given mixed mode occurs is correlated with the maximum value of the inner peak of the Brünt-Vaissälä frequency arising from a larger $\mu$ gradient. Such a mixed mode is detected in postZams model M20, M22, M23 and M24 in Fig. 8.

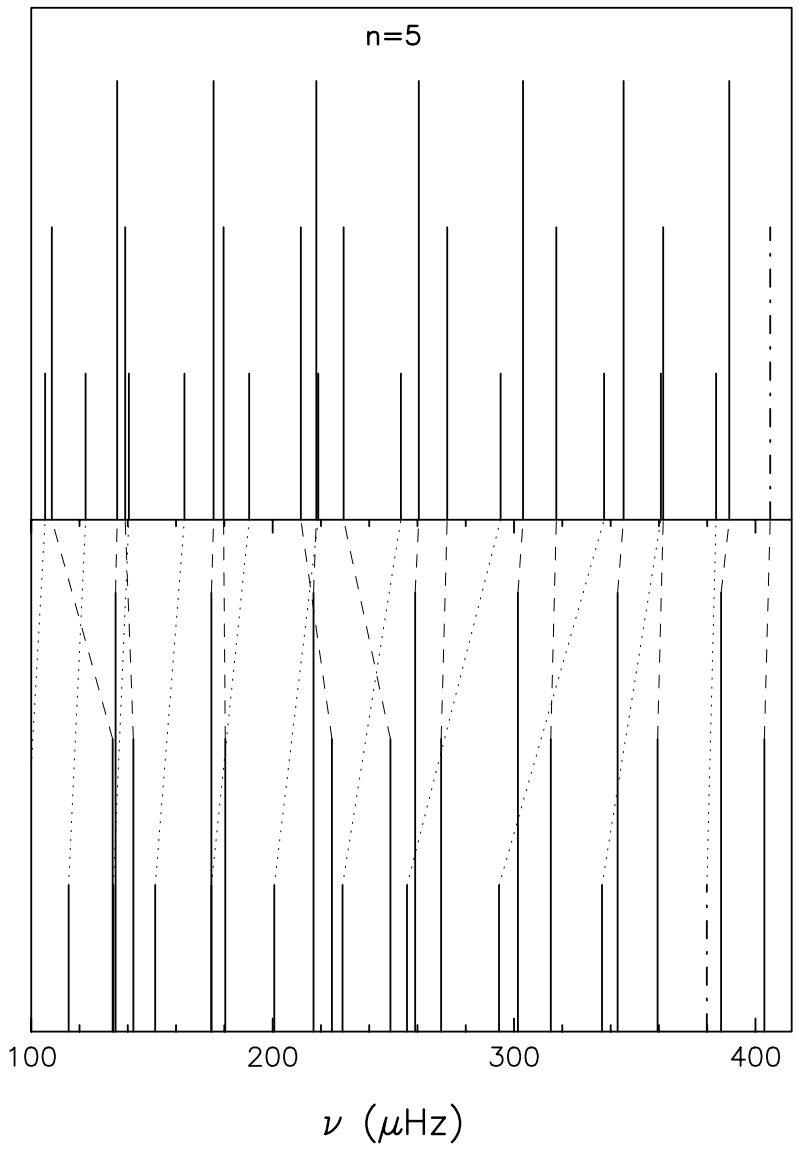

Fig. 6. Theoretical power spectra in the low frequency range where modes are excited. Top: postZams M23 model. Bottom: pms M4 model. Modes with same $(\ell, n)$ subscripts in both models are connected with dashed lines $(\ell=0, \ell=1$ modes) and dotted lines $(\ell=2)$ modes. Amplitudes are arbitrary. For clarity the highest amplitudes correspond to $\ell=0$ modes (thicker lines), medium ones to $\ell=1$ modes and smallest ones to $\ell=2$ modes. Marginally stable modes (modes with $-0.005 \leq \eta \leq 0)$ are pictured with dash-dotted vertical lines.

While the main sequence model ages, the progression of the mixed mode toward higher frequencies is clearly seen. The frequency of the extra mode with the highest frequency gives an indication of the age of the postZams star. On the other hand, pms models show a quasi constant large separation (in normalized units) with frequency.

Differences in the inner layers of pms and postZams models can also be detected by investigating the behavior of the small separations $\nu_{n, \ell}-\nu_{n^{\prime}, \ell-2}$ as a function of the frequency $\nu_{n, \ell}$. In the asymptotic regime i.e. for high radial order modes (solar like oscillations) the small separation is defined as $\nu_{n, \ell}-\nu_{n-1, \ell-2}$. In the low radial order range, avoided crossings perturb the regular sequence of alternations of $\ell=2$ and $\ell=0$ modes. We therefore find more convenient to define a small separation as the difference between a $\ell=2$ mode (radial order $n^{\prime}$ ) and its closest $\ell=0$ neighbor (radial order $n$ which in some cases at low frequency differs from $\left.n^{\prime}+1\right)$. Such small separations are plotted in Fig. 9. When the associated models are very close to the Zams as for instance models M13-M16, 


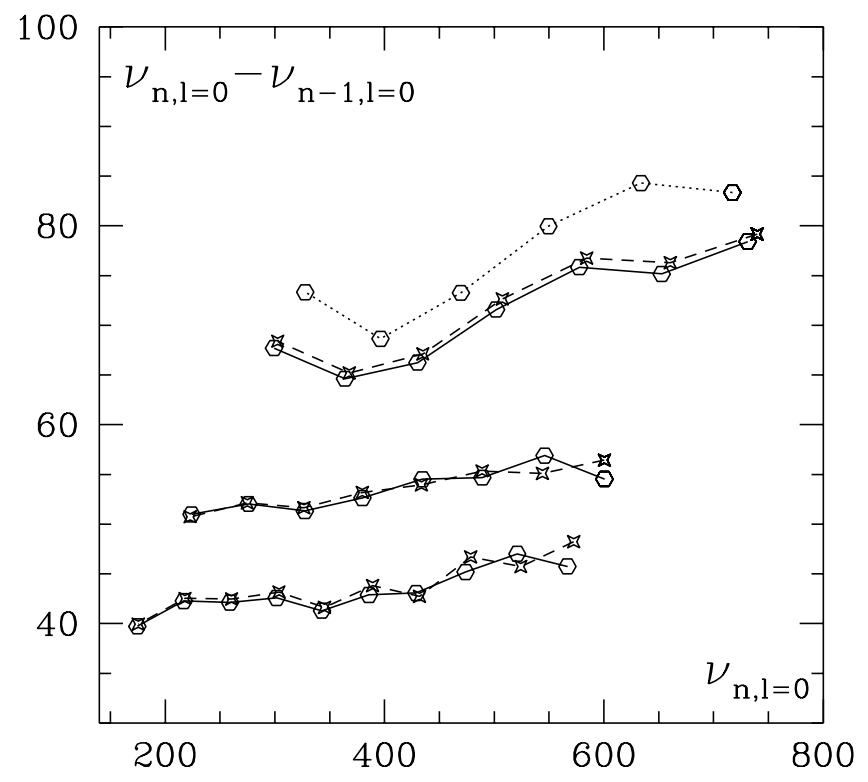

Fig. 7. Large separations $\nu_{n \ell}-\nu_{n-1, \ell}(\mu \mathrm{Hz})$ for low radial order $\ell=0$ modes for associated models from bottom to top M4M23; M8-M19; M13-M16 (pms: solid lines; postZams dashed lines) and the Zams model M15 (dotted line): large separations are the same for pms and postZams models with the same mean density.

no difference exists between the small separations of the models. For these models, as no $\mu$ gradient has developed yet, there is no avoided crossing in the excited frequency range.

On the other hand, for associated models located further away from the Zams, for instance M4-M23, the structures of the inner layers are significantly different; this leads to a different behavior of the small separations of the two models at low frequency. An avoided crossing is seen to occur for modes with $\nu \sim 350 \mu \mathrm{Hz}(\sigma \sim 8)$ for model M23 which is not present for its associated pms model M4 (Fig. 9). At lower frequencies (below $250 \mu \mathrm{Hz}$ corresponding to $\sigma \sim 6$ ), a second feature is seen for both models M4 and M23: this sharp variation of the small separation is due to modes with frequencies close to the value of the secondary inner maximum of the Brünt-Vaissälä frequency (the only inner maximum for pms models) near the edge of the convective core.

\section{Discussion}

Pre-main sequence models of 1.7-2 $M_{\odot}$ crossing the classical instability strip on their way to the Zams, are found to be vibrationally unstable, destabilized by the same mechanisms as the $\delta$ Scuti stars, as main sequence or postZams stars.

For a given mass and a given location in the HR diagram, the frequencies of radial modes with same radial order remain nearly identical. As the outer layers in both stages are almost the same, oscillations in pms stars should have the same level of amplitudes as in $\delta$ Scuti stars; this

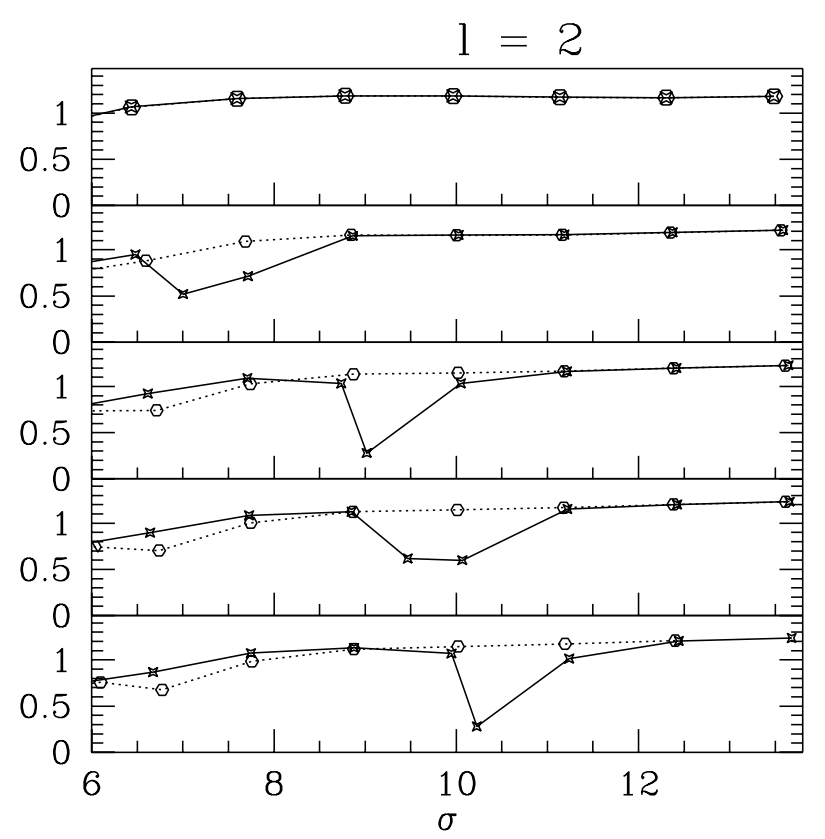

Fig. 8. Large separations $\sigma_{n, \ell}-\sigma_{n-1, \ell}$ for $\ell=2$ versus $\sigma_{n, \ell=2}$ for associated pms (dotted lines) and postZams (solid lines) models from top to bottom: M13-M16; M7-M20; M5-M22; M4-M23; M3-M24.

means that some modes are expected to be detectable from ground.

Low radial order modes with dual (gravity and pressure) nature penetrate in the upper layers of the star and there is a good chance to observe them. We have shown that they can act as discriminators, as they are very sensitive to the structure of the inner layers, which differ between pms and postZams stars. This is particularly interesting for this region of the HR diagram where classical indicators of youth as accretion disks or remaining nebulosities have disappeared.

Frequency shifts and differences in the frequency distributions of pms and main sequence oscillators can be detected if a sufficient number of modes are observed at low frequencies in the vinicity of the fundamental radial mode.

Important progress has been made in detecting larger sets of frequencies from the ground using networks of telescopes covering all longitudes. Nevertheless, ideal conditions will only be achieved in space. The COROT experiment (Baglin et al. 1998), to be launched in 2004, will continuously observe several stars over 20 to 150 days; this will provide an increase of a thousand for the signal to noise ratio and a frequency resolution from $0.5 \mu \mathrm{Hz}$ down to $0.1 \mu \mathrm{Hz}$. The large number of modes which is expected to be detected will help to provide, at least, a partial mode identification. Frequency histograms for instance can provide the mean density (Breger et al. 1999; Baglin et al. 2000; Barban et al. 2001) and the structure of the frequency spectrum will tell whether the star is a pms or a postZams star. 


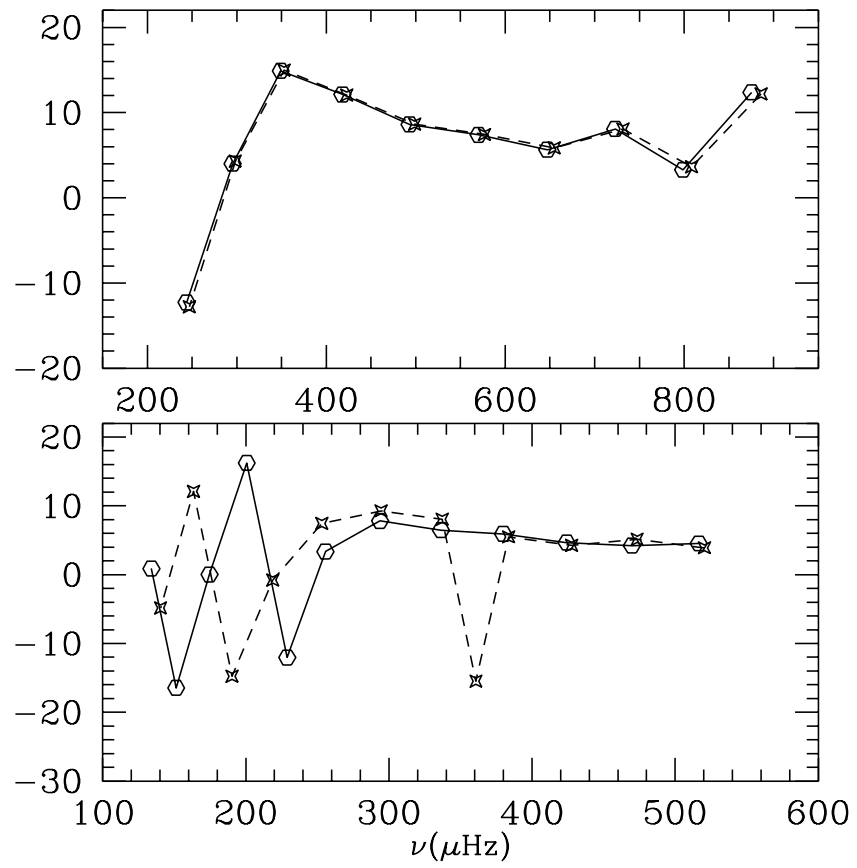

Fig. 9. Small separations $\nu_{n, \ell=0}-\nu_{n^{\prime}, \ell=2}$ versus $\nu_{n, \ell=2}$ (in $\left.\mu \mathrm{Hz}\right)$ for associated pms (solid lines, open circles) and postZams (dashed lines, open stars) models: M4-M23 (bottom) and M13-M16 (top). The frequency difference is calculated between a $\ell=2$ mode and the $\ell=0$ mode with the closest frequency.

Pms stars are expected to be fast rotators and it will be necessary to include effects of rotation on the frequencies in the same way as proposed by Soufi et al. (1998) for fast rotating $\delta$ Scuti stars. This should enable to put constraints on the internal rotation of these stars (Goupil et al. 1996). It will then be possible to trace the history and to better understand the process - of transfer of angular momentum inside stars with $\sim 2 M_{\odot}$ in their early stages of evolution.

Acknowledgements. We are grateful to P. Morel for making available the CESAM code. M. D. Suran acknowledges ParisMeudon Observatory for providing him a grant. We thank an anonymous referee for his comments which helped to improve the writing of this manuscript.

\section{References}

Alexander, D. R., \& Ferguson, J. W. 1994, ApJ, 437, 879
Baglin, A. and the COROT team 1998, IAU Symp. 185, ed. F. Deubner (Kluwer), 301

Baglin, A., Barban, C., Goupil, M. J., Michel, E., \& Auvergne, A. 2000, PASP, 210, in Delta Scuti and related stars, ed. M. Breger, \& M. H. Montgomery, 359

Barban, C., Goupil, M. J., Van'tVeer, C., \& Garrido, R. 2001, in Sogo 10/Gong 2000 workshop, ESA SP-464, 399

Breger, M., Pamyatnykh, A. A, Pikall, H., \& Garrido, R. 1999, A\&A, 341, 151

Buchler, J. R., Yecko, P. A., \& Kollath, Z. 1997, A\&A, 326, 669

Catala, C., Boehm, T., Donati, J.-F., et al. 1997, A\&A, 319, 176

Caughlan, G. R., \& Fowler, W. A. 1988, Atom. Data Nucl. Data Tables, 40, 284

Christensen-Dalsgaard, J., \& Berthomieu, G. 1992, in Solar interior and Atmosphere, ed. A. N. Cox, W. C. Livingston, \& M. S. Matthews (Univ. of Arizona Press, Tucson)

Cox, J. P. 1980, Theory of Stellar Pulsation (Princeton University Press)

Dziembowski, W. 1993, in IAU Symp. 137, ed. A. Baglin, \& W. Weiss, 521

Eggleton, P. P., Faulkner, J., \& Flannery, B. P. 1973, A\&A, 23,325

Fernandes, J., Lebreton, Y., \& Baglin, A. 1998, A\&A, 311, 127

Gautschy, A., \& Saio, H. 1996, ARA\&A, 34, 551

Goupil, M. J., Dziembovsky, W., Goode, P. R., \& Michel, E. 1996, A\&A, 305, 498

Goupil, M. J., Michel, E., Lebreton, Y., \& Baglin, A. 1993, A\&A, 268, 546

Grevesse, N., \& Noels, A. 1993, in Origin and Evolution of the Elements, ed. N. Prantzos, E. Vangioni-Flam, \& M. Casse (Cambridge University Press), 14

Iglesias, C., \& Rogers, F. 1996, ApJ, 464, 943

Kurtz, D. W., \& Marang, F. 1995, MNRAS, 276, 191

Kurtz, D. W. 2000, PASP, 210, in Delta Scuti and related stars, ed. M. Breger, \& M. H. Montgomery, 287

Marconi, M., \& Palla, F. 1998, ApJL, 507, 141

Morel, P. 1997, A\&AS, 124, 597

Saio, H., Baker, N. H., \& Gautschy, A. 1998, MNRAS, 294, 622

Saio, H., \& Cox, J. P. 1980, ApJ, 236, 549

Soufi, F., Goupil, M. J., \& Dziembowski, W. A. 1998, A\&A, 334,911

Suran, M. D. 1998, in IAU Symp. 185, ed. F. Deubner (Kluwer), 397

Unno, W., Osaki, Y., Ando, H., Saio, H., \& Shybahashi, H. 1989, Nonradial Oscillations of stars, 2nd Ed. (University of Tokyo Press) 